\title{
Metabolomic signatures of the long-term exposure to air pollution and temperature
}

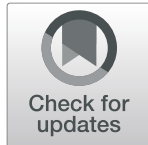

\author{
Feiby L. Nassan ${ }^{1 *}$ (D, Rachel S. Kelly ${ }^{2}$, Anna Kosheleva ${ }^{1}$, Petros Koutrakis ${ }^{1}$, Pantel S. Vokonas ${ }^{3}$, \\ Jessica A. Lasky-Su² and Joel D. Schwartz ${ }^{1,2,4}$
}

\begin{abstract}
Background: Long-term exposures to air pollution has been reported to be associated with inflammation and oxidative stress. However, the underlying metabolic mechanisms remain poorly understood.

Objectives: We aimed to determine the changes in the blood metabolome and thus the metabolic pathways associated with long-term exposure to outdoor air pollution and ambient temperature.
\end{abstract}

Methods: We quantified metabolites using mass-spectrometry based global untargeted metabolomic profiling of plasma samples among men from the Normative Aging Study (NAS). We estimated the association between longterm exposure to $\mathrm{PM}_{2.5}, \mathrm{NO}_{2}, \mathrm{O}_{3}$, and temperature (annual average of central site monitors) with metabolites and their associated metabolic pathways. We used multivariable linear mixed-effect regression models (LMEM) while simultaneously adjusting for the four exposures and potential confounding and correcting for multiple testing. As a reduction method for the intercorrelated metabolites (outcome), we further used an independent component analysis (ICA) and conducted LMEM with the same exposures.

Results: Men $(N=456)$ provided 648 blood samples between 2000 and 2016 in which 1158 metabolites were quantified. On average, men were 75.0 years and had an average body mass index of $27.7 \mathrm{~kg} / \mathrm{m}^{2}$. Almost all men (97\%) were not current smokers. The adjusted analysis showed statistically significant associations with several metabolites (58 metabolites with $\mathrm{PM}_{2.5}, 15$ metabolites with $\mathrm{NO}_{2}$, and 6 metabolites with temperature) while no metabolites were associated with $\mathrm{O}_{3}$. One out of five ICA factors (factor 2 ) was significantly associated with $\mathrm{PM}_{2.5}$. We identified eight perturbed metabolic pathways with long-term exposure to $\mathrm{PM}_{2.5}$ and temperature: glycerophospholipid, sphingolipid, glutathione, beta-alanine, propanoate, and purine metabolism, biosynthesis of unsaturated fatty acids, and taurine and hypotaurine metabolism. These pathways are related to inflammation, oxidative stress, immunity, and nucleic acid damage and repair.

Conclusions: Using a global untargeted metabolomic approach, we identified several significant metabolites and metabolic pathways associated with long-term exposure to $\mathrm{PM}_{2.5}, \mathrm{NO}_{2}$ and temperature. This study is the largest metabolomics study of long-term air pollution, to date, the first study to report a metabolomic signature of longterm temperature exposure, and the first to use ICA in the analysis of both.

Keywords: Metabolomics, Air pollution, Particulate matter, Temperature, Normative aging study (NAS)

\footnotetext{
*Correspondence: fen769@mail.harvard.edu

'Department of Environmental Health, Harvard T. H. Chan School of Public Health, Landmark Center, Room 414C, 401 Park Dr, Boston, MA 02215, USA

Full list of author information is available at the end of the article
}

C The Author(s). 2021 Open Access This article is licensed under a Creative Commons Attribution 4.0 International License, which permits use, sharing, adaptation, distribution and reproduction in any medium or format, as long as you give appropriate credit to the original author(s) and the source, provide a link to the Creative Commons licence, and indicate if changes were made. The images or other third party material in this article are included in the article's Creative Commons licence, unless indicated otherwise in a credit line to the material. If material is not included in the article's Creative Commons licence and your intended use is not permitted by statutory regulation or exceeds the permitted use, you will need to obtain permission directly from the copyright holder. To view a copy of this licence, visit http://creativecommons.org/licenses/by/4.0/. The Creative Commons Public Domain Dedication waiver (http://creativecommons.org/publicdomain/zero/1.0/) applies to the data made available in this article, unless otherwise stated in a credit line to the data. 


\section{Highlights}

1. Long-term air pollution exposure is associated with adverse outcomes.

2. The blood metabolome is a powerful tool for mechanistic understanding.

3. We identified metabolites and metabolic pathways that were perturbed with long-term exposure to $\mathrm{PM}_{2.5}$ and temperature.

4. Similar to short-term exposure, the perturbed pathways with long-term exposure are related to inflammation and oxidative stress, immunity, and nucleic acid damage and repair.

\section{Introduction}

Long-term exposures to air pollution [1-3] and temperature [4] have been reported to be associated with oxidative stress and inflammation. These biological processes are linked to mortality $[5,6]$ and several adverse health effects such as pulmonary [7], cardiovascular [8], and neurological diseases [9]. However, the affected metabolic mechanisms linking these exposures and phenotypes remain poorly understood. The metabolome is a collection of biologically active chemicals derived from the combination of endogenous processes and exogenous exposures [10]. Metabolomic profiling of blood is a powerful tool to increase mechanistic understanding. Because some ambient air pollutants e.g., fine particles, have been reported to enter the bloodstream directly from the lungs, a blood metabolomic signature associated with ambient air pollution exposure is plausible [11]. Even larger particles that cannot cross the pulmonary epithelium and ambient temperature can induce inflammation in the lungs and trigger a systemic response observed in the blood [12, 13].

Recently a few studies [14-19], including one (currently under consideration for publication elsewhere) based within the cohort studied here (The Normative Aging Study, NAS), have examined the metabolomic signatures of short-term exposure (days) to air pollution and, less frequently, short-term temperature [20]. However, to date, only three studies focused on long-term exposure to air pollution [21-23] and no human studies have examined the effects of long-term exposure to temperature, thus the mechanistic understanding is still lacking. Furthermore, a few studies have employed methods to capture the high dimensionality of the data and account for the intercorrelation of the metabolites within co-regulated pathways.

Furthermore, a key issue in the existing studies of temperature and mortality is that they do not merely identify increased deaths at the extremes of temperature [24]. They generally report U-shaped relationships with a minimum mortality temperature, typically in the range of $15-20^{\circ} \mathrm{C}$, with increased death rates at any other temperature [24]. For example, there is clearly increased mortality in Boston at $25^{\circ} \mathrm{C}$ compared to the minimum mortality temperature [24]. Furthermore, similar associations of non-extreme temperature exposure with other adverse health outcomes other than mortality were reported [25]. Hence it is critical to identify mechanisms by which temperature may have these effects. Changes in the metabolome are one such possible mechanism.

Therefore, we aimed in this study to determine the blood metabolomic signature of long-term exposure to outdoor air pollution and temperature, while using novel statistical methods e.g., independent component analysis (ICA) to reduce the dimensions of the intercorrelated data. Using Ultrahigh Performance Liquid Chromatography Coupled Tandem Mass Spectroscopy (UPLC-MS/MS) based plasma untargeted metabolomic profiling of a large cohort of men (NAS), we hypothesized that long-term (annual) exposure to air pollution and temperature are associated with perturbed biological pathways and corresponding blood metabolomic signatures. We further tested if there was an effect modification by metabolic conditions like obesity or diabetes.

\section{Materials and methods}

\section{Participants and study design}

The NAS was established in 1963 in Boston, Massachusetts. It is a longitudinal aging study among men. Men $(N=2280), 21-80$ years old and free of known chronic diseases were enrolled in the NAS between 1963 and 1970 and have been followed since [26]. The review boards of Harvard T.H. Chan School of Public Health and the Department of Veterans Affairs approved the NAS. All participants provided written informed consent. During the follow-up visits, participants periodically self-reported information about their medical history, dietary intake, and other health-related history. In addition, participants had physical examinations and laboratory tests every 3-5 years. At every visit, a $7 \mathrm{ml}$ fasting venous blood sample was collected in a trace metal-free tube containing ethylenediaminetetraacetic acid. The samples were spun for $15 \mathrm{~min}$ at 3000 revolutions per minute (RPM). Serum and plasma samples were placed in $1.8 \mathrm{ml}$ Nunc tubes for long-term storage at $-80^{\circ} \mathrm{C}$.

For the current analysis, we considered 464 men who provided 659 blood samples between 2000 and 2016 in which blood metabolomics profiling was performed. Blood samples collected prior to 2000 were not suitable for metabolomic profiling due to their storage conditions. Because only eight men were non-white, we restricted our final sample size to 456 white men who provided 648 blood samples. 


\section{Quantification of air pollution and temperature (exposure)}

In the current study, we focused on long-term exposures to air pollution and temperature as measured by the annual average (365 days) of the levels before the visit for each blood draw. The air pollutants were particulate matter $\leq 2.5 \mu \mathrm{m}\left(\mathrm{PM}_{2.5}\right)$ and gaseous pollutants: nitrogen dioxide $\left(\mathrm{NO}_{2}\right)$ and ozone $\left(\mathrm{O}_{3}\right)$. All exposures were measured near downtown Boston, MA, at a fixed monitoring site at the Harvard University Countway Library and $\sim 1$ $\mathrm{km}$ from the NAS examination center. Because study participants lived in the greater Boston area with a median distance of $\sim 20.8 \mathrm{~km}$ from the examination center [27], we considered the ambient air pollutant levels as participants' long-term exposure surrogates. Levels of $\mathrm{PM}_{2.5}\left(\mu \mathrm{g} / \mathrm{m}^{3}\right)$ were measured hourly using a tapered element oscillation microbalance (Model 1400A, Rupprecht and Pastashnick) [28]. Hourly $\mathrm{NO}_{2}$ and $\mathrm{O}_{3}$ levels (part per billion (ppb)) were measured at local Massachusetts Department of Environmental Protection monitoring sites in the greater Boston area and were averaged based on data from all available sites. We obtained daily temperature and relative humidity data from the national weather service station at Logan airport (Boston, MA), located $\sim 12 \mathrm{~km}$ from the NAS examination site. Study participants lived throughout the metropolitan area. Therefore, we considered the monitored temperature as surrogates of their long-term exposures.

\section{Metabolomic profiling (outcomes)}

All samples were sent to the lab and analyzed at the same timepoint. Metabolon Inc. (Durham, NC, USA) conducted metabolomic profiling. To enable the broadest coverage of the metabolome, untargeted highresolution UPLC-MS/MS was used. The methods were previously described in detail [29]. In brief, the four platforms were: (1) UPLC-MS/MS under positive ionization for early eluting metabolites, (2) UPLC-MS/MS under positive ionization for late eluting metabolites, (3) UPLC-MS/MS under negative ionization, and (4) UPLCMS/MS, polar (negative ionization) platform. The samples were randomized for profiling and in-house standards and internal QCs were used to account for potential batch effects across the runs [29]. Metabolites were identified by their mass-to-charge ratio, retention time, and through a comparison to a library of purified known standards. Metabolites were quantified using area-under-the-curve (AUC) of the peak and processed according to our in-house standard quality control pipeline $[30,31]$ which retains the maximum number of metabolites, including those with a high level of missingness as these may represent biologically important markers of exposure in a subset of the population, while excluding statistically uninformative metabolites. Missing values were imputed with half of the minimum observed level for a given metabolite [31]. Then, metabolite levels were log-transformed and pareto scaled. A total of 1301 metabolites were profiled including 143 metabolites with an interquartile range of zero that we considered uninformative and hence excluded. This left 1158 metabolites available for the current analysis.

\section{Statistical analysis}

We calculated descriptive statistics to summarize demographics and lifestyle factors for the study participants during the first visit as well as all visits. We examined whether the annual averages of the levels of $\mathrm{PM}_{2.5}, \mathrm{NO}_{2}$, $\mathrm{O}_{3}$, and temperature prior to visit were associated with changes in the levels of the 1158 metabolites. To account for the correlation of repeated measures within the same participant, we used linear mixed-effect regression models (LMEM) with random participant-specific intercepts. We first conducted generalized additive mixed models (GAMM) with penalized spline for temperature to check if linearity would fit better, then we used LMEM as our final models because temperature fit better as a linear term. We modeled the outcomes and the exposures as continuous variables. We simultaneously adjusted for $\mathrm{PM}_{2.5}, \mathrm{NO}_{2}, \mathrm{O}_{3}$, and temperature (multi-pollutant models) as shown below. We further adjusted for potential confounders and predictors of the outcomes including age (years), body mass index $(\mathrm{kg} /$ $\mathrm{m}^{2}$ ), socioeconomic status (annual income and years of education), cigarette pack-years, alcohol intake $(<$ or $\geq 2$ drinks per day), season at blood draw (warm/cold), and relative humidity.

Multi-pollutant models:

$$
\begin{aligned}
E\left(Y_{i j}\right)= & \beta_{0}+\mu_{i}+\beta_{1} \text { PM }_{2.5 i j}+\beta_{2} N_{2} O_{2 i j}+\beta_{3} O_{3 i j} \\
& +\beta_{4} \text { Temperature }_{i j}+\beta_{5-n} \text { Covariates }_{i j}
\end{aligned}
$$

where $Y_{i j}$ is the metabolome level of subject $\mathrm{i}$ at visit $\mathrm{j}$, $\beta_{0}$ is the fixed intercept, $\mu_{i}$ is the random intercept for subject $i$, and the annual averages of the air pollutants and temperature for subject $i$ at visit $j$.

To correct for multiple testing while also accounting for the highly-correlated metabolites that are closely connected through interlinked biological pathways, we conducted the number of the effective tests approach (ENT) $[32,33]$. This method determines the number of principal components required to explain a given percentage of the variance in the data (i.e., the number of effective/independent tests). We calculated the adjusted $p$-value threshold as $\alpha / m$ where $\alpha$ is the nominal $p$-value of 0.05 , and $m$ is the number of principal components. We used thresholds of 95\% (ENT95\%) and 99\% (ENT99\%) variance explained and further divided it by 
four (investigated exposures). We finally tested for effect modification by type II diabetes and obesity (BMI $\geq 30$ $\mathrm{kg} / \mathrm{m}^{2}$ ) for the four exposures by adding interaction terms for every metabolite.

In order to explore metabolomic profiles rather than individual metabolites, we also applied independent component analysis (ICA) [34] as an unsupervised technique to reduce the dimension of the highly-correlated metabolites into five independent ICA-factors. The more commonly used principal component analysis (PCA) is a tool for dividing multiple correlated variables into uncorrelated factors. If the variables are normally distributed then uncorrelated factors are also independent, however that is not true for non-normally distributed variables such as metabolites here. In contrast, ICA is a computational method for separating multivariate signal into additive factors that are maximally independent that assumes non-normal signals [34]. There is an attached weight for each metabolite to determine its individual contributing weight to each ICA-factor. We then conducted LMEM where those five ICA independent factors were the outcomes while exposures and covariates remained as above. We further corrected for multiple testing in the ICA models by applying BenjaminiHochberg false discovery rate (FDR), set the false positive threshold as 5\% [35], and further accounted for the four investigated exposures $\left(\mathrm{PM}_{2.5}, \mathrm{NO}_{2}, \mathrm{O}_{3}\right.$, and temperature).

As a sensitivity analysis, we conducted LMEM models for each single pollutant or temperature at a time (singlepollutant models) as shown below, while adjusting for the covariates as above (but not other exposures). The singlepollutant models for air pollutants were still simultaneously adjusted for temperature. Analyses were conducted using $\mathrm{R}$ version 4.0.1 and SAS version 9.4 (SAS Institute Inc., Cary, NC, USA).

Single - pollutant models:

$$
\begin{aligned}
E\left(Y_{i j}\right)= & \beta_{0}+\mu_{i}+\beta_{1} \text { PM }_{2.5 i j}+\beta_{2} \text { Temperature }_{i j} \\
& +\beta_{3-n} \text { Covariates }_{i j} \\
E\left(Y_{i j}\right)= & \beta_{0}+\mu_{i}+\beta_{1} N_{2 i j}+\beta_{2} \text { Temperature }_{i j} \\
& +\beta_{3-n} \text { Covariates }_{i j} \\
E\left(Y_{i j}\right)= & \beta_{0}+\mu_{i}+\beta_{1} O_{3 i j}+\beta_{2} \text { Temperature }_{i j} \\
& +\beta_{3-n} \text { Covariates }_{i j} \\
E\left(Y_{i j}\right)= & \beta_{0}+\mu_{i}+\beta_{1} \text { Temperature }_{i j} \\
& +\beta_{3-n} \text { Covariates }_{i j}
\end{aligned}
$$

As an exploratory analysis to better understand the significant metabolites, we conducted pathway analysis for the significant metabolites (at $p$-value $<0.01$ ) from the multi-pollutant model and the 100 metabolites that had the greatest weighting on the significant ICA- factor(s). To do that, we used the 'Pathway Analysis' functionality in MetaboAnalyst 4.0, that accounts for both over-representation (i.e., how many significant metabolites fall within a given pathway) and pathway topology (i.e., how important those metabolites are to that pathway) [36] using Human Metabolome Database (HMDB), and Kyoto Encyclopedia of Genes and Genomes (KEGG) databases. The structure of metabolic pathways provides information on the complex relationships between molecules (activation, inhibition, or reaction, etc.). The pathway topology analyses incorporates this information by using two well-established node centrality measures to estimate the importance of a given metabolite within a pathway 1) betweenness centrality which focuses on the position of the metabolite relative to overall pathway structure and 2) degree centrality which focuses on how connected that metabolite is to other metabolites within the pathway [37]. The importance of each metabolite is defined as the percentage in relation to the total pathway importance, and the pathway impact is defined as the cumulative percentage from the matched metabolites [37].

We considered statistical significance for the pathways at a nominal $p$-value $\leq 0.1$ and considered additional noteworthy pathways if the impact score was $\geq 0.5$ while nominal $p$-value $<0.3$.

\section{Results}

Participants $(N=456)$ provided 648 blood samples in which 1158 distinct metabolites were quantified and passed our in-house QC pipeline [30, 31]. Approximately $64 \%$ of the participants provided one blood sample, $31 \%$ provided two samples, and $6 \%$ provided three samples. The measured metabolites constituted lipids (39\%), followed by metabolites of unidentified origin (19\%), amino acids (17\%), xenobiotics $(12 \%)$, nucleotides $(3 \%)$, cofactor and vitamins $(3 \%)$, carbohydrates $(2 \%)$, peptides $(2 \%)$, partially characterized molecules (2\%), and energy metabolites (1\%). At the initial visit, men's mean age was $\sim 75.0$ years, mean body mass index was $27.7 \mathrm{~kg} / \mathrm{m}^{2}$, mean annual income was 8.61 thousand of 1965 \$US, and participants had received a mean of 15.1 years of education (Table 1). Almost all participants (97\%) were not current smokers. Most of the participants (79\%) consumed $<2$ alcoholic drinks/day. Approximately $60 \%$ of the visits occurred during the warm season (April to September). Average values of air pollution and temperature over the followup period remained stable across the 365-day exposure window (Table 1).

After adjusting for potential confounding and correcting for multiple testing in the multi-pollutant models, $\mathrm{PM}_{2.5}, \mathrm{NO}_{2}$, and temperature showed statistically significant associations with several metabolites (58 
Table 1 Characteristics of the study population in the Normative Aging Study (2000 to 2016)

\begin{tabular}{|c|c|c|}
\hline Demographic Characteristics & At first visit (2000 to 2013$)(N=456)$ & All visits (2000 to 2016$)(N=648)$ \\
\hline Age, Mean (SD) & $75.0(6.70)$ & $76.0(6.68)$ \\
\hline Body mass index $\left(\mathrm{kg} / \mathrm{m}^{2}\right)$, Mean (SD) & $27.7(4.19)$ & $27.7(4.27)$ \\
\hline Years of education, Mean (SD) & $15.1(3.00)$ & $15.0(3.00)$ \\
\hline Baseline annual income, thousands of $1965 \$ U S$, Mean (SD) & $8.61(3.77)$ & $8.64(3.78)$ \\
\hline \multicolumn{3}{|l|}{ Smoking } \\
\hline Never, N (\%) & $128(28)$ & $190(29)$ \\
\hline Current, N (\%) & $14(3)$ & $21(3)$ \\
\hline Former, N (\%) & $314(69)$ & $437(67)$ \\
\hline Pack-year smoked (years), Mean (SD) & $21.4(25.2)$ & $20.95(25.36)$ \\
\hline \multicolumn{3}{|l|}{ Season } \\
\hline Cold: October-March, N (\%) & $189(41)$ & $259(40)$ \\
\hline Warm: April-September, N (\%) & $267(59)$ & $389(60)$ \\
\hline Alcohol consumption ( $\geq 2$ drinks per day), N (\%) & $95(21)$ & $132(20)$ \\
\hline \multicolumn{3}{|l|}{ Mean (SD), (IQR) of the exposure levels } \\
\hline Average of 365 days of $\mathrm{PM}_{2.5}(\mu \mathrm{g} / \mathrm{m} 3)$ & $10.4(1.28),(2.49)$ & $10.1(1.31),(2.12)$ \\
\hline Average of 365 days of $\mathrm{NO}_{2}$ (ppb) & $19.1(2.40),(4.52)$ & $18.4(2.35),(4.00)$ \\
\hline Average of 365 days of $\mathrm{O}_{3}(\mathrm{ppb})$ & $23.7(1.06),(1.39)$ & $24.0(1.12),(1.45)$ \\
\hline Average of 365 days of Temperature $\left({ }^{\circ} \mathrm{C}\right)$ & $11.0(0.64),(1.00)$ & $11.0(0.60),(0.89)$ \\
\hline
\end{tabular}

Abbreviations: $N$ Number of participants or visits, SD Standard deviation, $p p b$ is part per billion, Kg Kilogram, $m$ Meters, C Celsius, IQR Interquartile range

metabolites with $\mathrm{PM}_{2.5}, 15$ metabolites with $\mathrm{NO}_{2}$, and 6 metabolites with temperature) out of 1158 metabolites while no metabolites were associated with $\mathrm{O}_{3}$ (Fig. 1, Tables 2 and 3, Supplemental Table 1). It is worth mentioning that several significant metabolites were unidentified at the time of this analysis, and annotation is pending. On the other hand, heptanoate (7:0), inosine, and guanosine were among the identified ones, for example with ambient temperature exposure, with negative associations (Table 3). In the ICA analysis, in the multi-pollutant models, $\mathrm{PM}_{2.5}$ was significantly associated with ICA-factor 2 only but not with $\mathrm{NO}_{2}$ or $\mathrm{O}_{3}$ (Supplemental Table 2). Many of the most contributing metabolites (highest 100) to ICA-factor 2 were also individually significant with exposures of interest in the multi-pollutant models (Tables 2 and 3, Supplemental Table 1). We did not observe an effect modification by diabetes or obesity for any of the examined exposures on any of the metabolites examined at the ENT95\% level. Results were similar in the sensitivity analysis of the single-pollutant models, but in the latter analysis a larger number of metabolites were significantly associated with each outcome (Supplemental Figure 1 and Supplemental Table 3).

In the exploratory pathway analysis of the significant metabolites for each exposure, long-term exposure to $\mathrm{PM}_{2.5}$ was associated with five metabolic pathways including: glycerophospholipid ( $p$-value $=0.009)$, propanoate $(p$-value $=0.03)$, sphingolipid $(p$-value $=0.04)$, and glutathione $(p$-value $=0.12)$ metabolism $($ Fig. 2 and Supplemental Table 4). Long-term exposure to $\mathrm{NO}_{2}$ was not significantly associated with any metabolic pathways. Long-term exposure to temperature was associated with biosynthesis of unsaturated fatty acids $(p$-value $=0.01)$. The pathways that were associated with the 100 highest contributing metabolites to ICA-factor 2 were purine $(p$-value $=0.02), \quad$ sphingolipid $(p$-value $=0.03)$, beta-alanine $(p$-value $=0.06)$, and glycerophospholipid $(p$-value $=0.12)$ metabolism. Taurine and hypotaurine metabolism was also noteworthy due to high impact score of 0.71 while $p$-value was 0.27 .

\section{Discussion}

Using an untargeted metabolomic approach, we observed several significant associations between longterm air pollutants $\left(\mathrm{PM}_{2.5}\right.$ and $\left.\mathrm{NO}_{2}\right)$ and temperature with several blood metabolites. We identified eight metabolic pathways perturbed with long-term exposure to $\mathrm{PM}_{2.5}$ and temperature: glycerophospholipid, sphingolipid, glutathione, beta-alanine, propanoate, purine metabolisms, biosynthesis of unsaturated fatty acids, and possibly taurine and hypotaurine metabolism. In this study, we assessed the largest number of metabolites using an untargeted approach and the largest sample size to date in studies of metabolite association with long-term air pollution. This is also the first study to report a metabolomic signature of long-term temperature exposure, and the first to use ICA in the 


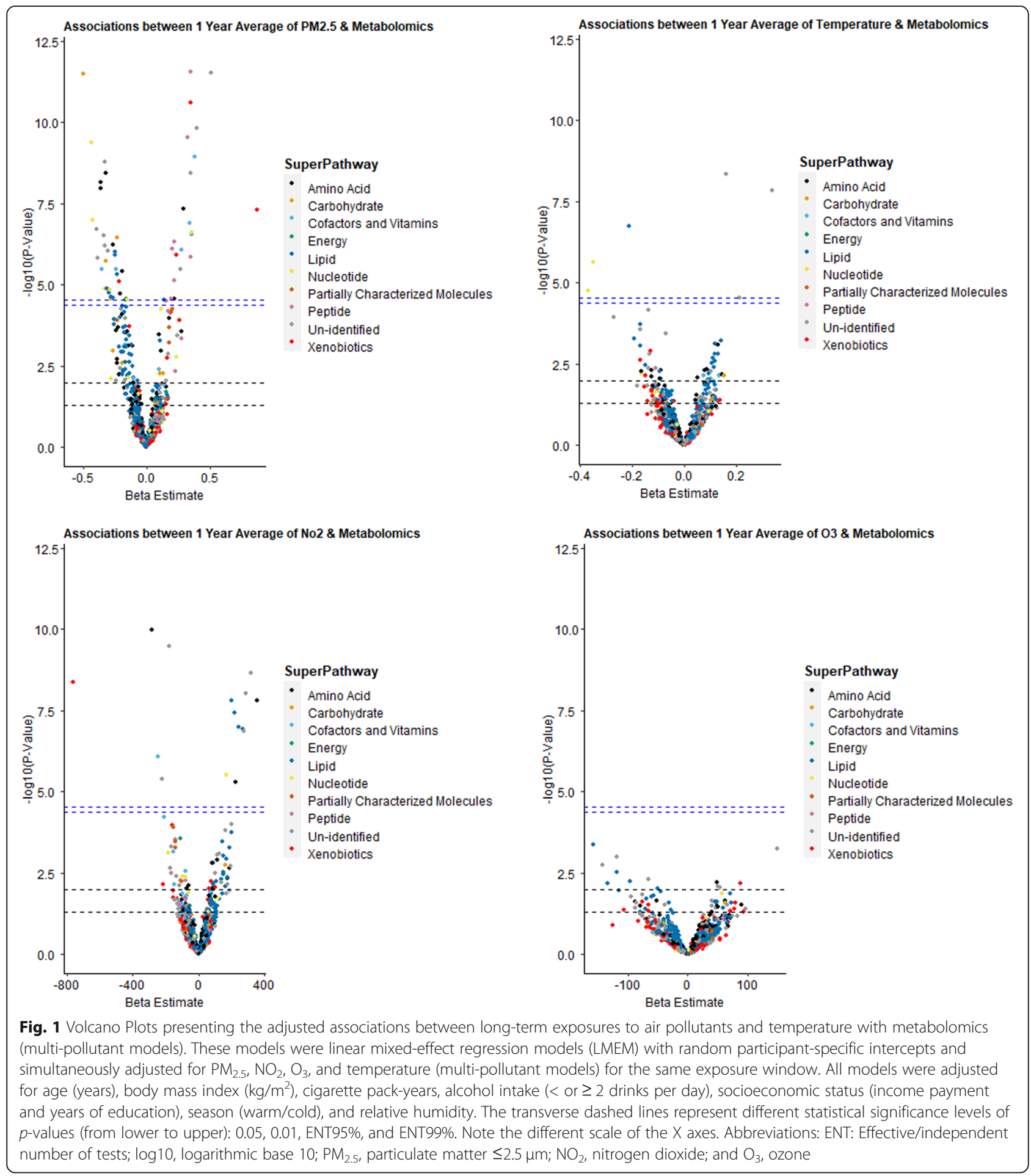

analysis of both. There was an overlap between the perturbed pathways when we used different statistical methods i.e., regression models and ICA. Previously some studies [38, 39] used PCA to analyze metabolomics data which produces uncorrelated factors, a necessary but insufficient condition for independence. In comparison ICA is a computational method to separate multivariate signals into additive maximally independent factors and assumes non-normal signals [34].

For example, with ambient temperature, heptanoate (7:0), inosine, and guanosine were negatively associated with ambient temperature exposure. Heptanoate $(7: 0)$ or Heptanoic acid is a straight medium-chain saturated fatty acid that contributes to the odor of some rancid 
Table 2 The significant adjusted associations between long-term (annual) exposure to $\mathrm{PM}_{2.5}$ with metabolomics (multi-pollutant models) at ENT95\% significance level

\begin{tabular}{|c|c|c|c|c|c|c|}
\hline BIOCHEMICAL & Super-Pathway & Sub-Pathway & $\begin{array}{l}\text { Beta (SE) for } \\
\text { individual } \\
\text { metabolites }\end{array}$ & $\begin{array}{l}P \text {-value for } \\
\text { individual } \\
\text { metabolites }\end{array}$ & $\begin{array}{l}\text { ICA_factor2 } \\
\text { Weight }\end{array}$ & $\begin{array}{l}\text { ICA } \\
\text { factor2 } \\
\text { Rank }\end{array}$ \\
\hline Aspartate & Amino Acid & Alanine and Aspartate Metabolism & $-0.203(0.046)$ & $1.93 \mathrm{E}-05$ & 0.392 & 57 \\
\hline Cys-Gly, Oxidized & Amino Acid & Glutathione Metabolism & $-0.357(0.060)$ & 1.10E-08 & 0.527 & 19 \\
\hline Cysteinylglycine Disulfide $^{*}$ & Amino Acid & Glutathione Metabolism & $-0.316(0.051)$ & 4.00E-09 & 0.434 & 43 \\
\hline Cysteinylglycine & Amino Acid & Glutathione Metabolism & $-0.361(0.059)$ & 7.00E-09 & 0.266 & 115 \\
\hline Sarcosine & Amino Acid & Glycine, Serine and Threonine Metabolism & $0.293(0.051)$ & 4.60E-08 & 0.094 & 291 \\
\hline Alpha-Hydroxyisocaproate & Amino Acid & Leucine, Isoleucine and Valine Metabolism & $-0.266(0.051)$ & 5.69E-07 & 0.483 & 27 \\
\hline Taurine & Amino Acid & $\begin{array}{l}\text { Methionine, Cysteine, SAM and Taurine } \\
\text { Metabolism }\end{array}$ & $-0.190(0.040)$ & 3.79E-06 & 0.423 & 45 \\
\hline Cysteine S-Sulfate & Amino Acid & $\begin{array}{l}\text { Methionine, Cysteine, SAM and Taurine } \\
\text { Metabolism }\end{array}$ & $-0.580(0.075)$ & $6.76 \mathrm{E}-13$ & 0.412 & 50 \\
\hline $\begin{array}{l}\text { 5-Methylthioadenosine } \\
\text { (MTA) }\end{array}$ & Amino Acid & Polyamine Metabolism & $0.222(0.052)$ & 2.77E-05 & -0.277 & 108 \\
\hline Maltose & Carbohydrate & Glycogen Metabolism & $-0.321(0.065)$ & $1.90 \mathrm{E}-06$ & 0.536 & 17 \\
\hline 3-Phosphoglycerate & Carbohydrate & $\begin{array}{l}\text { Glycolysis, Gluconeogenesis, and Pyruvate } \\
\text { Metabolism }\end{array}$ & $-0.499(0.067)$ & $3.33 \mathrm{E}-12$ & 0.856 & 2 \\
\hline Lactate & Carbohydrate & $\begin{array}{l}\text { Glycolysis, Gluconeogenesis, and Pyruvate } \\
\text { Metabolism }\end{array}$ & $-0.233(0.044)$ & $3.54 \mathrm{E}-07$ & 0.448 & 39 \\
\hline Ribitol & Carbohydrate & Pentose Metabolism & $-0.278(0.065)$ & $2.68 \mathrm{E}-05$ & 0.204 & 152 \\
\hline Heme & $\begin{array}{l}\text { Cofactors and } \\
\text { Vitamins }\end{array}$ & Hemoglobin and Porphyrin Metabolism & $-0.348(0.073)$ & $3.34 \mathrm{E}-06$ & 0.599 & 10 \\
\hline Bilirubin $(E, Z \text { Or } Z, E)^{*}$ & $\begin{array}{l}\text { Cofactors and } \\
\text { Vitamins }\end{array}$ & Hemoglobin and Porphyrin Metabolism & $0.354(0.066)$ & $2.93 \mathrm{E}-07$ & -0.327 & 79 \\
\hline Bilirubin $(E, E)^{*}$ & $\begin{array}{l}\text { Cofactors and } \\
\text { Vitamins }\end{array}$ & Hemoglobin and Porphyrin Metabolism & $0.343(0.062)$ & $1.30 \mathrm{E}-07$ & -0.260 & 118 \\
\hline Bilirubin $(Z, Z)$ & $\begin{array}{l}\text { Cofactors and } \\
\text { Vitamins }\end{array}$ & Hemoglobin and Porphyrin Metabolism & $0.381(0.059)$ & $1.00 \mathrm{E}-09$ & -0.117 & 243 \\
\hline Nicotinamide & $\begin{array}{l}\text { Cofactors and } \\
\text { Vitamins }\end{array}$ & Nicotinate and Nicotinamide Metabolism & $-0.237(0.049)$ & $3.27 \mathrm{E}-06$ & 0.515 & 22 \\
\hline Alpha-Tocopherol & $\begin{array}{l}\text { Cofactors and } \\
\text { Vitamins }\end{array}$ & Tocopherol Metabolism & $0.279(0.055)$ & 8.57E-07 & -0.121 & 233 \\
\hline Malate & Energy & TCA Cycle & $-0.164(0.038)$ & 2.93E-05 & 0.311 & 90 \\
\hline Succinate & Energy & TCA Cycle & $-0.161(0.037)$ & $2.72 \mathrm{E}-05$ & 0.249 & 127 \\
\hline 1-Stearoyl-GPS (18:0) & Lipid & Lysophospholipid & $-0.31(0.069)$ & $1.30 \mathrm{E}-05$ & 0.640 & 7 \\
\hline 1-Arachidonoyl-GPA (20:4) & Lipid & Lysophospholipid & $-0.282(0.065)$ & $2.40 \mathrm{E}-05$ & 0.462 & 34 \\
\hline $\begin{array}{l}\text { 1,2-Dipalmitoyl-GPE } \\
(16: 0 / 16: 0)^{*}\end{array}$ & Lipid & Phosphatidylethanolamine (PE) & $-0.299(0.068)$ & $1.79 \mathrm{E}-05$ & 0.406 & 52 \\
\hline $\begin{array}{l}\text { 1-Stearoyl-2-Oleoyl-GPS } \\
\text { (18:0/18:1) }\end{array}$ & Lipid & Phosphatidylserine (PS) & $-0.264(0.061)$ & $2.55 \mathrm{E}-05$ & 0.640 & 8 \\
\hline Phosphoethanolamine & Lipid & Phospholipid Metabolism & $-0.228(0.049)$ & $4.92 \mathrm{E}-06$ & 0.552 & 14 \\
\hline Sphinganine & Lipid & Sphingolipid Synthesis & $-0.249(0.049)$ & $9.96 \mathrm{E}-07$ & 0.530 & 18 \\
\hline $\begin{array}{l}\text { Sphingomyelin } \\
(\text { D18:2/18:1) }\end{array}$ & Lipid & Sphingomyelins & $0.137(0.032)$ & $2.88 \mathrm{E}-05$ & -0.066 & 423 \\
\hline Sphingosine & Lipid & Sphingosines & $-0.249(0.049)$ & $1.17 \mathrm{E}-06$ & 0.562 & 13 \\
\hline $\begin{array}{l}\text { Inosine 5'-Monophosphate } \\
\text { (IMP) }\end{array}$ & Nucleotide & $\begin{array}{l}\text { Purine Metabolism, (Hypo)Xanthine/ } \\
\text { Inosine containing }\end{array}$ & $-0.423(0.076)$ & $1.02 \mathrm{E}-07$ & 0.799 & 3 \\
\hline Hypoxanthine & Nucleotide & $\begin{array}{l}\text { Purine Metabolism, (Hypo)Xanthine/ } \\
\text { Inosine containing }\end{array}$ & $-0.327(0.073)$ & $1.33 \mathrm{E}-05$ & 0.755 & 5 \\
\hline
\end{tabular}


Table 2 The significant adjusted associations between long-term (annual) exposure to $\mathrm{PM}_{2.5}$ with metabolomics (multi-pollutant models) at ENT95\% significance level (Continued)

\begin{tabular}{|c|c|c|c|c|c|c|}
\hline BIOCHEMICAL & Super-Pathway & Sub-Pathway & $\begin{array}{l}\text { Beta (SE) for } \\
\text { individual } \\
\text { metabolites }\end{array}$ & $\begin{array}{l}P \text {-value for } \\
\text { individual } \\
\text { metabolites }\end{array}$ & $\begin{array}{l}\text { ICA_factor2 } \\
\text { Weight }\end{array}$ & $\begin{array}{l}\text { ICA_ } \\
\text { factor2 } \\
\text { Rank }\end{array}$ \\
\hline Allantoin & Nucleotide & $\begin{array}{l}\text { Purine Metabolism, (Hypo)Xanthine/ } \\
\text { Inosine containing }\end{array}$ & $-0.229(0.043)$ & $3.45 \mathrm{E}-07$ & 0.012 & 1007 \\
\hline $\begin{array}{l}\text { Adenosine } \\
\text { 5'-Monophosphate (AMP) }\end{array}$ & Nucleotide & Purine Metabolism, Adenine containing & $-0.433(0.066)$ & 4.16E-10 & 0.797 & 4 \\
\hline Dihydroorotate & Nucleotide & Pyrimidine Metabolism, Orotate containing & $0.356(0.066)$ & $2.41 \mathrm{E}-07$ & -0.279 & 106 \\
\hline Uracil & Nucleotide & Pyrimidine Metabolism, Uracil containing & $-0.284(0.064)$ & $1.40 \mathrm{E}-05$ & 0.399 & 56 \\
\hline Beta-Alanine & Nucleotide & Pyrimidine Metabolism, Uracil containing & $-0.149(0.035)$ & 2.87E-05 & 0.214 & 147 \\
\hline Gamma-Glutamylmethionine & Peptide & Gamma-glutamyl Amino Acid & $0.324(0.049)$ & $2.84 \mathrm{E}-10$ & -0.384 & 60 \\
\hline Gamma-Glutamylalanine & Peptide & Gamma-glutamyl Amino Acid & $0.347(0.07)$ & $1.41 \mathrm{E}-06$ & -0.360 & 65 \\
\hline Gamma-Glutamylglycine & Peptide & Gamma-glutamyl Amino Acid & $0.35(0.047)$ & $2.76 \mathrm{E}-12$ & -0.290 & 100 \\
\hline Gamma-Glutamylglutamine & Peptide & Gamma-glutamyl Amino Acid & $0.22(0.042)$ & 4.59E-07 & -0.250 & 125 \\
\hline Gamma-Glutamylvaline & Peptide & Gamma-glutamyl Amino Acid & $0.216(0.047)$ & 7.33E-06 & -0.229 & 138 \\
\hline Gamma-Glutamylisoleucine* & Peptide & Gamma-glutamyl Amino Acid & $0.197(0.046)$ & 2.64E-05 & -0.205 & 151 \\
\hline $\begin{array}{l}\text { Gamma-Glutamyl-Alpha- } \\
\text { Lysine }\end{array}$ & Peptide & Gamma-glutamyl Amino Acid & $0.207(0.04)$ & 7.56E-07 & -0.192 & 162 \\
\hline Gamma-Glutamylthreonine & Peptide & Gamma-glutamyl Amino Acid & $0.152(0.036)$ & $3.03 \mathrm{E}-05$ & -0.077 & 351 \\
\hline Iminodiacetate (IDA) & Xenobiotics & Chemical & $0.352(0.049)$ & $2.51 \mathrm{E}-11$ & -0.125 & 227 \\
\hline Hepes & Xenobiotics & Chemical & $0.874(0.154)$ & 4.90E-08 & 0.062 & 447 \\
\hline $\begin{array}{l}\text { Tartronate } \\
\text { (Hydroxymalonate) }\end{array}$ & Xenobiotics & Food Component/Plant & $0.238(0.047)$ & 1.19E-06 & -0.442 & 41 \\
\hline Ergothioneine & Xenobiotics & Food Component/Plant & $-0.213(0.046)$ & 7.84E-06 & 0.268 & 112 \\
\hline$x-24,970$ & Un-identified & - & $-0.389(0.072)$ & 2.01E-07 & 0.360 & 63 \\
\hline$X-24,307$ & Un-identified & - & $0.512(0.068)$ & $3.06 \mathrm{E}-12$ & -0.340 & 74 \\
\hline$x-23,739$ & Un-identified & - & $0.266(0.056)$ & $3.36 \mathrm{E}-06$ & -0.337 & 76 \\
\hline$X-12,104$ & Un-identified & - & $0.349(0.056)$ & 4.00E-09 & -0.319 & 84 \\
\hline$x-11,632$ & Un-identified & - & $-0.329(0.052)$ & 2.00E-09 & 0.318 & 85 \\
\hline$x-13,684$ & Un-identified & - & $-0.383(0.077)$ & 1.55E-06 & 0.317 & 86 \\
\hline$X-24,306$ & Un-identified & - & $0.397(0.059)$ & 1.57E-10 & -0.165 & 184 \\
\hline$X-24,431$ & Un-identified & - & $-0.334(0.063)$ & $3.10 \mathrm{E}-07$ & 0.160 & 190 \\
\hline$X-14,904$ & Un-identified & - & $-0.327(0.063)$ & $6.26 \mathrm{E}-07$ & -0.119 & 237 \\
\hline$X-10,458$ & Un-identified & - & $-0.303(0.059)$ & 8.90E-07 & 0.022 & 865 \\
\hline
\end{tabular}

These models were linear mixed-effect regression models (LMEM) with random participant-specific intercepts and simultaneously adjusted for $\mathrm{PM}_{2.5}, \mathrm{NO}_{2}, \mathrm{O}_{3}$, and temperature (multi-pollutant models) for the same exposure window

Beta (SE) and $p$-values presented are from individual metabolites analysis

All models were adjusted for age (years), body mass index $\left(\mathrm{kg} / \mathrm{m}^{2}\right)$, cigarette pack-years, alcohol intake ( $<$ or $\geq 2$ drinks per day), socioeconomic status (income payment and years of education), season (warm/cold), and relative humidity

Significant metabolites at ENT95\% significance level only are presented

ICA_factor2 rank represents the rank of the corresponding metabolite contributing to factor 2 from the independent component analysis (ICA). Higher rank (and higher weights) means higher contribution to factor 2 of the ICA. We show alongside the weights and ranks of each metabolite that contributes to factor 2 from the ICA only because it was the only significant factor

Range of facto2- ICA rank is from 1 to 1158 i.e., number of examined metabolites. Range of facto2- ICA weight is from -1 to 1

The metabolites with an X-XXXX format are unknown (to date Metabolon has not been able to name the metabolite) but reproducible (Metabolon is reliably able to characterize this metabolite in multiple samples and studies)

Abbreviations: $E N T$ Effective/independent number of tests, $P M_{2.5}$ Particulate matter $\leq 2.5 \mu \mathrm{m}, \mathrm{NO}_{2}$ Nitrogen dioxide, and $\mathrm{O}_{3}$ Ozone, ICA Independent component analysis, SE Standard error

*Indicates a compound that has not been confirmed based on a standard, but Metabolon is confident in its identity based on a subset of these analytical parameters 
Table 3 The significant adjusted associations between long-term exposure windows to temperature with metabolomics (multipollutant models) at ENT95\% significance level

\begin{tabular}{|c|c|c|c|c|c|c|}
\hline Biochemical & $\begin{array}{l}\text { Super- } \\
\text { Pathway }\end{array}$ & Sub-Pathway & $\begin{array}{l}\text { Beta (SE) for individual } \\
\text { metabolites }\end{array}$ & $\begin{array}{l}P \text {-value for individual } \\
\text { metabolites }\end{array}$ & $\begin{array}{l}\text { ICA_factor2 } \\
\text { Weight }\end{array}$ & $\begin{array}{l}\text { ICA_factor2 } \\
\text { Rank }\end{array}$ \\
\hline Heptanoate (7:0) & Lipid & Medium Chain Fatty Acid & $-0.209(0.039)$ & 1.87E-07 & -0.282 & 104 \\
\hline Inosine & Nucleotide & $\begin{array}{l}\text { Purine Metabolism, (Hypo)Xanthine/ } \\
\text { Inosine containing }\end{array}$ & $-0.368(0.083)$ & $1.71 \mathrm{E}-05$ & 0.892 & 1 \\
\hline Guanosine & Nucleotide & $\begin{array}{l}\text { Purine Metabolism, Guanine } \\
\text { containing }\end{array}$ & $-0.348(0.071)$ & $2.23 \mathrm{E}-06$ & 0.329 & 78 \\
\hline$X-24,970$ & Un-identified & - & $0.34(0.057)$ & 1.50E-08 & 0.360 & 63 \\
\hline$x-24,431$ & Un-identified & - & $0.215(0.05)$ & 2.91E-05 & 0.160 & 190 \\
\hline$x-23,636$ & Un-identified & - & $0.161(0.026)$ & 5.00E-09 & 0.141 & 214 \\
\hline
\end{tabular}

These models were linear mixed-effect regression models (LMEM) with random participant-specific intercepts and simultaneously adjusted for $\mathrm{PM}_{2.5}, \mathrm{NO}_{2}, \mathrm{O}_{3}$, and temperature (multi-pollutant models) for the same exposure window

Beta (SE) and $p$-values presented are from individual metabolites analysis

All models were adjusted for age (years), body mass index $\left(\mathrm{kg} / \mathrm{m}^{2}\right)$, cigarette pack-years, alcohol intake $(<$ or $\geq 2$ drinks per day), socioeconomic status (income payment and years of education), season (warm/cold), and relative humidity

Significant metabolites at ENT95\% significance level only are presented

ICA_factor2 rank represents the rank of the corresponding metabolite contributing to factor 2 from the independent component analysis (ICA). Higher rank (and higher weights) means higher contribution to factor 2 of the ICA. We show alongside the weights and ranks of each metabolite that contributes to factor 2 from the ICA only because it was the only significant factor

Range of facto2- ICA rank is from 1 to 1158 i.e., number of examined metabolites. Range of facto2- ICA weight is from -1 to 1

The metabolites with an X-XXXX format are unknown (to date Metabolon has not been able to name the metabolite) but reproducible (Metabolon is reliably able to characterize this metabolite in multiple samples and studies)

Abbreviations: $E N T$ Effective/independent number of tests, $P M_{2.5}$ Particulate matter $\leq 2.5 \mu \mathrm{m}, \mathrm{NO}_{2}$ Nitrogen dioxide, and $\mathrm{O}_{3}$ Ozone, ICA Independent component analysis, SE Standard error, NS Not significant at the ENT95\% level

oils and used in the preparation of esters for the fragrance industry, and as an additive in cigarettes. It has a role as a plant metabolite [40]. It is capable of donating a hydron to an acceptor however little is known about health effects. On the other hand, guanosine and inosine are part of purine metabolism and have known antioxidant activity [41]. They have shown protection of DNA in-vitro from oxidative damage induced by reactive oxygen species (ROS) and served as radioprotectors in mice [41]. The negative association of ambient temperature exposure and those metabolites may indicate the potential vulnerability to oxidative stress by long-term exposure to ambient temperature.

Long-term exposures to air pollution [1-3] and temperature [4] have been linked to oxidative stress and inflammation. The identified pathways associated with long-term exposure to $\mathrm{PM}_{2.5}$ and temperature, similar to short-term exposure, are related to inflammation, oxidative stress, immunity, and nucleic acid damage and repair. Air pollutants may act directly as oxidants or generate free radicals that can cause oxidative stress [42]. One primary target of the reactive oxygen species derived from air pollutants is the cell membrane. Oxidative stress activates phospholipase A2 which then hydrolyzes phospholipids from the cell membrane to generate polyunsaturated free fatty acid and lyso-phospholipid [43, 44]. Due to the existence of double carbon bonds, the resulting fatty acids can be subsequently oxidized by oxygenase [44] and form products that are potential biomarkers for oxidative stress affecting lipids [45].
In our study, we observed associations with perturbed glycerophospholipid metabolism and unsaturated fatty acids biosynthesis that are main components of biological membranes as well as downstream products from oxidation of the membranes, respectively [43, 44]. This is consistent with our finding with short-term air pollution (will be reported elsewhere). Also, unsaturated fatty acids e.g., linolenic acid has been identified in association with air long-term air pollution exposure (mainly ultrafine particle (UFP)) that mediated adult onset asthma and cardio-cerebrovascular diseases [23]. We also observed perturbations in sphingolipid metabolism that is involved in inflammation and immunity $[46,47]$ consistent with finding with short-term air pollution [48]. Sphingolipid is also implicated in membrane biology [49] and has been linked to diabetes, hepatocellular carcinoma, and Alzheimer's disease [50, 51], heart failure [52], and cancer [47]. We observed perturbations in glutathione metabolism, involved in xenobiotic-mediated oxidative stress $[53,54]$, which has been reported to be associated with traffic-related air pollution [55]. We observed perturbations in the metabolism of sulfurcontaining amino acids, taurine and hypotaurine, which are readily oxidized [56]. This finding is consistent with exposure to short-term air pollution. In addition, consistent with findings with short-term air pollution, we observed perturbations in propanoate metabolism that is involved in branched-chain amino acids metabolism and the short-chain fatty acid metabolism [57]. Propanoate metabolism is involved in the colon microbiome and 


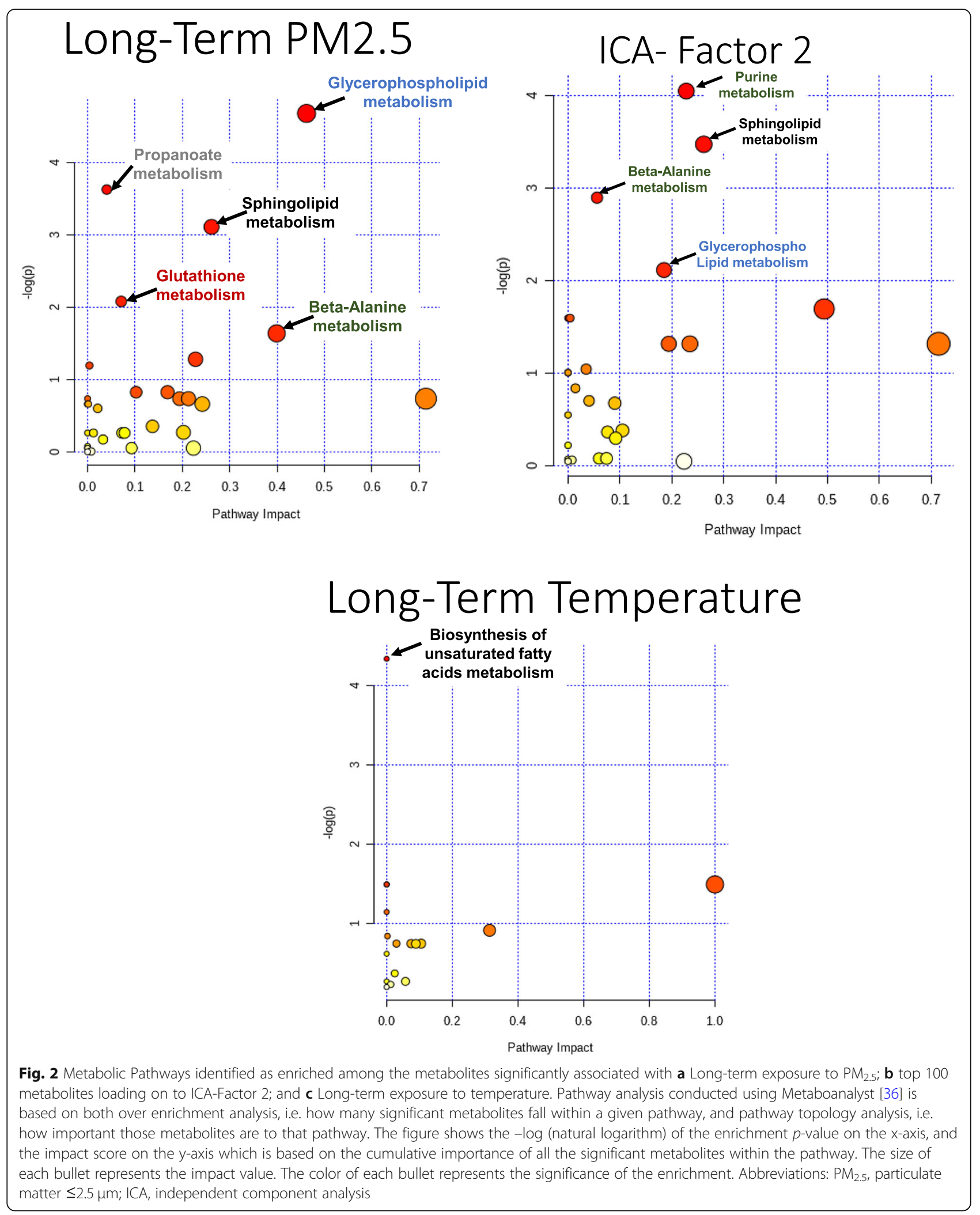


gluconeogenesis in the liver and acetate [58]. We observed perturbations in purine and beta-alanine metabolisms that are related to nucleic acid metabolism, damage and repair [59] consistent with reported association with air pollution, including with increased levels of 8-OHdG [19,60]. Those metabolic signatures could be potential biomarkers for long-term exposure to air pollution and temperature. Our results need to be replicated and validated in future studies to identify the specific pathways and metabolites perturbed by air pollution and temperature exposure and thus promising ways for prevention and therapy. Our results with temperature exposure could explain our previously reported excess mortality with both high and low temperature exposure that was not explained by heat [61].

To date, only three studies focused on long-term air pollution exposure. A cross-sectional study, among a subset $(N=523)$ of the TwinsUK cohort quantified untargeted metabolomic profiling (280 metabolites) [21]. The study reported that oxidative stress and inflammation related metabolites such as $\alpha$-tocopherol, glycine, and benzoate were associated with long-term $\mathrm{PM}_{2.5}$ and lung functions [21]. Another cross-sectional study of healthy participants $(N=59)$ measured 79 metabolites and reported associations between exposure to yearlong UFP with metabolic variations related to antioxidant pathways and endothelial function [22]. An additional publication that used data from Italy and Switzerland reported that metabolic pathways e.g., Linoleate metabolism, an unsaturated fatty acid, mediated the long-term effect of air pollution on adult onset asthma and cardiovascular disease [23]. Nevertheless, our results were generally consistent with the previous literature. All other studies had smaller sample size. None of the previous studies have used ICA in the analysis, which is a new analytical approach for high dimensional and intercorrelated data and none had coadjusted for ambient temperature. To the best of our knowledge, no studies have examined the association with long-term exposure to temperature on the metabolome.

Our results for long-term exposure to $\mathrm{PM}_{2.5}$ and temperature are consistent with our findings with shortterm exposure in the same study population (will be reported elsewhere). Short- and long-term exposure to $\mathrm{PM}_{2.5}$ was associated with unique 40 , and 58 metabolites, respectively and five similar perturbed metabolic pathways. On the other hand, long-term exposure to temperature was associated with six significant metabolites consistent with perturbed biosynthesis of unsaturated fatty acids, while short-term temperature exposure was associated with 40 significant metabolites and four perturbed metabolic pathways. We observed that long-term exposure to $\mathrm{NO}_{2}$ was associated with 15 unique significant metabolites but without significant perturbed metabolic pathways. However, short-term exposure to $\mathrm{NO}_{2}$ was significantly associated with 100 unique metabolites and four perturbed metabolic pathways (glycerophospholipid, glutathione, beta-alanine, and taurine and hypotaurine metabolisms). In addition, long-term exposure to $\mathrm{O}_{3}$ was not significantly associated with any metabolites, while short-term exposure to $\mathrm{O}_{3}$ was significantly associated with cysteine, which is a component of methionine, cysteine, SAM and taurine metabolism.

Our study had limitations including that a central site was used to assess the annual averages of the levels of outdoor air pollutants and temperature, which may differ from personal exposure. However, this potential misclassification may have led to underestimation of the association of interest instead of creating spurious ones $[62,63]$. We acknowledge the limitation of the exposure assessment in this study, however because this is the first study to explore this association, the results will need to be confirmed or refuted in future studies. Future studies would still benefit from the insight the study may provide given the big sample size. Our study participants were older white men who lived in the greater Boston area that is a low-polluted environment that may limit the generalizability of the results. The pathway analysis was exploratory with its inherent limitation of not taking the effect direction into account. Instead, pathway analysis is based on over representation and pathway topography i.e., position of the metabolite within pathway (if it is a hub metabolite or not). However, since metabolism is complex process with feedback loops, any effects on the pathway regardless of the direction is considered perturbation and worth further investigation. The results will need to be replicated in other areas and among women and more diverse population. In this all-male study, we can only speculate on the sex differences that have been reported in the effect of temperature and less consistently on effect of air pollution. Women have more subcutaneous fat than men and this could play a role in how temperature could affect metabolome. Hormonal changes especially post-menopausal also could matter in similar ways that could affect their risks for other disease e.g. cardiovascular disease.

Finally, because the NAS is an observational study, residual confounding cannot be excluded. However, we adjusted for several confounders. In addition, the results were reassuringly consistent when using different statistical methods. Our study had also a few strengths including that the NAS study participants were geographically stable and well followed-up since they were enrolled with $>80 \%$ response rates. In addition, we quantified the largest number of untargeted metabolites to date in association with long- 
term air pollution that increased the statistical power. We used an untargeted global approach to quantify metabolomics which has the potential to increase metabolomic coverage and to reduce bias towards identifying well-studied metabolites [16]. To the best to our knowledge, this is the first study to report a metabolomic signature of long-term temperature exposure. Finally, we used several rigorous statistical methods including the first use of ICA for metabolomic data and we also conducted pathway analysis to facilitate the mechanistic understanding and translation of the results.

\section{Conclusions}

Using an untargeted metabolomic approach, we identified several significant metabolites and metabolic pathways associated with long-term exposure to $\mathrm{PM}_{2.5}$ and temperature. We quantified the largest number of untargeted metabolites to date in association with long-term air pollution, first study to report a metabolomic signature of long-term temperature exposure, and the first to use ICA in the analysis of both. In this study, we provided insights about the metabolomic signatures of the long- and previously the short-term exposure to air pollution and temperature and the associated perturbation of the metabolic pathways. Global and untargeted metabolomic profiling is a powerful approach to increase the mechanistic understanding and the future biomarkers. Current developments of approaches for the annotation of metabolic pathways identified in the untargeted approach of the blood metabolome will allow future studies to delve deeper into the identity of specific metabolites that are affected by air pollution and temperature exposures. Our results should be replicated and validated in other studies of both shortand long-term effects of air pollution and temperature on human health. It is important in the future to identify the metabolomic signatures of the different components of $\mathrm{PM}_{2.5}$ to be able to identify the exposure sources.

\section{Supplementary Information}

The online version contains supplementary material available at https://doi. org/10.1186/s12940-020-00683-x.

Additional file 1.

\section{Acknowledgments}

We acknowledge all members of the Normative Aging Study (NAS) team. A special thanks to all study participants.

\section{Authors' contributions}

Conceptualization (FN, RK, JL-S, JS), Data curation (FN, RK, AK, PV, JS), Formal analysis (FN, JS), Funding acquisition (RK, PK, PV, JS), Investigation (FN, RK, JL$\mathrm{S}, J S)$, Methodology (FN, RK, JS), Project administration (FN, AK, JS), Resources (FN, RK, PV, JS), Software (FN, JS), Supervision (JS), Validation (FN, JS),
Visualization (FN), Writing - original draft (FN), Writing - review \& editing (FN, RK, AK, PK, PV, JL-S, JS). The author(s) read and approved the final manuscript.

\section{Funding}

This work was supported by U.S. EPA grant RD-835872 and NIEHSES01517201. This metabolomics profiling was supported by PR161204 W81XWH-17-10533 from the Congressionally Directed Medical Research Programs (CDMR P), USAMRDC. The VA Normative Aging Study is a research component of the Massachusetts Veterans Epidemiology Research and Information Center (MAVERIC) at VA Boston Healthcare System and is supported by the Cooperative Studies Program/Epidemiology Research and Information Centers, Office of Research and Development, US Department of Veterans Affairs. R.S.K was supported by K01HL146980 from the NHLBI. JLS was supported by R01HL123915 and R01HL141826 from NHLBI.

\section{Availability of data and materials}

The datasets used and/or analyzed during the current study are available from the corresponding author on reasonable request.

Ethics approval and consent to participate

The review boards of Harvard T.H. Chan School of Public Health and the Department of Veterans Affairs approved the NAS. All participants provided written informed consent.

Consent for publication

Not applicable.

\section{Competing interests}

The authors declare that they have no known competing financial interests or personal relationships that could have appeared to influence the work reported in this paper.

\section{Author details}

'Department of Environmental Health, Harvard T. H. Chan School of Public Health, Landmark Center, Room 414C, 401 Park Dr, Boston, MA 02215, USA. ${ }^{2}$ Channing Division of Network Medicine; Brigham and Women's Hospital, Harvard Medical School, Boston, MA 02129, USA. ${ }^{3}$ VA Normative Aging Study, VA Boston Healthcare System, School of Medicine and School of Public Health, Boston University, Boston, MA 02215, USA. ${ }^{4}$ Department of Epidemiology, Harvard T. H. Chan School of Public Health, Boston, MA 02115, USA.

Received: 5 August 2020 Accepted: 1 December 2020

Published online: 07 January 2021

\section{References}

1. Chen JC, Schwartz J. Metabolic syndrome and inflammatory responses to long-term particulate air pollutants. Environ Health Perspect. 2008;116(5):612-7.

2. Nwanaji-Enwerem JC, Wang W, Nwanaji-Enwerem O, et al. Association of Long-term Ambient Black Carbon Exposure and Oxidative Stress Allelic Variants with Intraocular Pressure in older men. JAMA Ophthalmol. 2019; 137(2):129-37.

3. Panasevich S, Leander K, Rosenlund $\mathrm{M}$, et al. Associations of long- and short-term air pollution exposure with markers of inflammation and coagulation in a population sample. Occup Environ Med. 2009;66(11):747-53.

4. Bind MA, Zanobetti A, Gasparrini A, et al. Effects of temperature and relative humidity on DNA methylation. Epidemiology. 2014:25(4):561-9.

5. Armstrong B, Sera F, Vicedo-Cabrera AM, et al. The role of humidity in associations of high temperature with mortality: a multicountry, multicity study. Environ Health Perspect. 2019;127(9):97007.

6. Wang Y, Shi L, Lee M, et al. Long-term Exposure to PM2.5 and Mortality Among Older Adults in the Southeastern US. Epidemiology (Cambridge, Mass). 2017;28(2):207-14.

7. Rice $\mathrm{MB}$, Ljungman PL, Wilker EH, et al. Long-term exposure to traffic emissions and fine particulate matter and lung function decline in the Framingham heart study. Am J Respir Crit Care Med. 2015;191(6):656-64.

8. Ljungman PLS, Li W, Rice MB, et al. Long- and short-term air pollution exposure and measures of arterial stiffness in the Framingham heart study. Environ Int. 2018;121(Pt 1):139-47. 
9. Bejot $Y$, Reis J, Giroud M, Feigin V. A review of epidemiological research on stroke and dementia and exposure to air pollution. Int J Stroke. 2018;13(7): 687-95.

10. Rappaport SM, Barupal DK, Wishart D, Vineis P, Scalbert A. The blood Exposome and its role in discovering causes of disease. Environ Health Perspect. 2014;122(8):769-74.

11. Nemmar A, Hoet PHM, Vanquickenborne B, et al. Passage of inhaled particles into the blood circulation in humans. Circulation. 2002;105(4):411-4.

12. Hogg JC, van Eeden S. Pulmonary and systemic response to atmospheric pollution. Respirology. 2009;14(3):336-46.

13. Halonen Jl, Zanobetti A, Sparrow D, Vokonas PS, Schwartz J. Associations between outdoor temperature and markers of inflammation: a cohort study. Environ Health. 2010;9:42.

14. Ward-Caviness CK, Breitner S, Wolf K, et al. Short-term NO2 exposure is associated with long-chain fatty acids in prospective cohorts from Augsburg, Germany: results from an analysis of 138 metabolites and three exposures. Int J Epidemiol. 2016;45(5):1528-38.

15. Li H, Cai J, Chen R, et al. Particulate matter exposure and stress hormone levels: a randomized, double-blind, Crossover Trial of Air Purification. Circulation. 2017;136(7):618-27.

16. Vlaanderen JJ, Janssen NA, Hoek G, et al. The impact of ambient air pollution on the human blood metabolome. Environ Res. 2017;156:341-8.

17. van Veldhoven $K$, Kiss A, Keski-Rahkonen $P$, et al. Impact of short-term traffic-related air pollution on the metabolome - results from two metabolome-wide experimental studies. Environ Int. 2019;123:124-31.

18. Surowiec I, Karimpour M, Gouveia-Figueira S, et al. Multi-platform metabolomics assays for human lung lavage fluids in an air pollution exposure study. Anal Bioanal Chem. 2016;408(17):4751-64.

19. Liang D, Moutinho JL, Golan R, et al. Use of high-resolution metabolomics for the identification of metabolic signals associated with traffic-related air pollution. Environ Int. 2018;120:145-54.

20. Hampel R, Breitner S, Kraus WE, et al. Short-term effects of air temperature on plasma metabolite concentrations in patients undergoing cardiac catheterization. Environ Res. 2016;151:224-32.

21. Menni C, Metrustry SJ, Mohney RP, et al. Circulating levels of antioxidant vitamins correlate with better lung function and reduced exposure to ambient pollution. Am J Respir Crit Care Med. 2015;191(10):1203-7.

22. Walker DI, Lane KJ, Liu K, et al. Metabolomic assessment of exposure to nearhighway ultrafine particles. J Expo Sci Environ Epidemiol. 2019;29(4):469-83.

23. Jeong $A$, Fiorito $G$, Keski-Rahkonen $P$, et al. Perturbation of metabolic pathways mediates the association of air pollutants with asthma and cardiovascular diseases. Environ Int. 2018;119:334-45.

24. Gasparrini A, Guo Y, Hashizume M, et al. Mortality risk attributable to high and low ambient temperature: a multicountry observational study. Lancet 2015;386(9991):369-75

25. Zanobetti A, Coull BA, Kloog I, et al. Fine-scale spatial and temporal variation in temperature and arrhythmia episodes in the VA normative aging study. J Air Waste Manag Assoc. 2017;67(1):96-104.

26. Payton M, Riggs KM, Spiro A 3rd, Weiss ST, Hu H. Relations of bone and blood lead to cognitive function: the VA normative aging study. Neurotoxicol Teratol. 1998;20(1):19-27.

27. Lepeule J, Bind M-AC, Baccarelli AA, et al. Epigenetic influences on associations between air pollutants and lung function in elderly men: the normative aging study. Environ Health Perspect. 2014;122(6):566-72.

28. Koutrakis P, Sioutas C, Ferguson ST, Wolfson JM, Mulik JD, Burton RM. Development and evaluation of a glass honeycomb denuder/filter pack system to collect atmospheric gases and particles. Environ Sci Technol. 1993;27(12):2497-501.

29. Evans AB, Mitchell M, Liu Q, Stewart S, Dai H, Dehaven C, Miller L. High Resolution Mass Spectrometry Improves Data Quantity and Quality as Compared to Unit Mass Resolution Mass Spectrometry in High-Throughput Profiling Metabolomics. Metabolomics. 2014;4(132):1

30. Kelly RS, Chawes BL, Blighe K, et al. An integrative Transcriptomic and Metabolomic study of lung function in children with asthma. Chest. 2018;154(2):335-48.

31. Kelly RS, Bayne H, Spiro A 2nd, et al. Metabolomic signatures of lead exposure in the VA normative aging study. Environ Res. 2020;190:110022.

32. Li MX, Yeung JM, Cherny SS, Sham PC. Evaluating the effective numbers of independent tests and significant $p$-value thresholds in commercial genotyping arrays and public imputation reference datasets. Hum Genet. 2012;131(5):747-56.

33. Nyholt DR. A simple correction for multiple testing for single-nucleotide polymorphisms in linkage disequilibrium with each other. Am J Hum Genet. 2004;74(4):765-9.
34. Adali T, Levin-Schwartz Y, Calhoun VD. Multi-modal data fusion using source separation: two effective models based on ICA and IVA and their properties. Proc IEEE Inst Electr Electron Eng. 2015;103(9):1478-93.

35. Benjamini $Y$, Hochberg $Y$. Controlling the false discovery rate: a practical and powerful approach to multiple testing. J R Stat Soc Ser B Methodol. 1995;57(1):289300.

36. Chong J, Wishart DS, Xia J. Using MetaboAnalyst 4.0 for Comprehensive and Integrative Metabolomics Data Analysis. Curr Protoc Bioinformatics. 2019;68(1):e86.

37. Xia J, Wishart DS. MetPA: a web-based metabolomics tool for pathway analysis and visualization. Bioinformatics (Oxford, England). 2010;26(18):2342-4.

38. Zhang C, Zhao B, Zhang C, et al. Mechanisms of bergenin treatment on chronic bronchitis analyzed by liquid chromatography-tandem mass spectrometry based on metabolomics. Biomed Pharmacother. 2019;109:2270-7.

39. Ning L, Shan G, Sun Z, et al. Serum proteome profiles to differentiate Crohn disease from intestinal tuberculosis and primary intestinal lymphoma: a pilot study. Medicine (Baltimore). 2019;98(50):e18304.

40. PubChem. PubChem Compound Summary for CID 8094, Heptanoic acid. 2020 https:/pubchem.ncbi.nlm.nih.gov/compound/Heptanoic-acid. Accessed $21 \mathrm{Oct}$ 2020.

41. Gudkov SV, Shtarkman IN, Smirnova VS, Chernikov AV, Bruskov VI. Guanosine and inosine display antioxidant activity, protect DNA in vitro from oxidative damage induced by reactive oxygen species, and serve as radioprotectors in mice. Radiat Res. 2006;165(5):538-45.

42. Kelly FJ. Oxidative stress: its role in air pollution and adverse health effects. Occup Environ Med. 2003:60(8):612-6.

43. Anthonymuthu TS, Kenny EM, Lamade AM, Kagan VE, Bayır H. Oxidized phospholipid signaling in traumatic brain injury. Free Radic Biol Med. 2018; 124:493-503.

44. Sato H, Taketomi Y, Murakami M. Metabolic regulation by secreted phospholipase A2. Inflamm Regen. 2016;36(1):7.

45. Tam Vincent C, Quehenberger O, Oshansky Christine M, et al. Lipidomic profiling of influenza infection identifies mediators that induce and resolve inflammation. Cell. 2013;154(1):213-27.

46. Hannun YA, Obeid LM. Sphingolipids and their metabolism in physiology and disease. Nat Rev Mol Cell Biol. 2018;19(3):175-91.

47. Voelkel-Johnson C, Norris JS, White-Gilbertson S. Chapter Ten - Interdiction of Sphingolipid Metabolism Revisited: Focus on Prostate Cancer. In: Chalfant CE, Fisher PB, editors. Advances in Cancer Research, vol. 140: Academic Press; 2018 p. 265-93. https://www.ncbi.n/m.nih.gov/pmc/articles/PMC6460930/.

48. Yan Q, Liew Z, Uppal K, et al. Maternal serum metabolome and trafficrelated air pollution exposure in pregnancy. Environ Int. 2019;130:104872.

49. Gault CR, Obeid LM, Hannun YA. An overview of sphingolipid metabolism: from synthesis to breakdown. Adv Exp Med Biol. 2010;688:1-23.

50. Pralhada Rao R, Vaidyanathan N, Rengasamy M, Mammen Oommen A Somaiya N, Jagannath MR. Sphingolipid metabolic pathway: an overview of major roles played in human diseases. J Lipids. 2013;2013: 178910

51. Pujol-Lereis LM. Alteration of Sphingolipids in Biofluids: Implications for Neurodegenerative Diseases. Int J Mol Sci. 2019;20(14):3564.

52. Contaifer D Jr, Buckley LF, Wohlford G, et al. Metabolic modulation predicts heart failure tests performance. PLoS One. 2019;14(6):e0218153.

53. Gonzalez FJ. Role of cytochromes P450 in chemical toxicity and oxidative stress: studies with CYP2E1. Mutat Res. 2005;569(1-2):101-10.

54. Meister A. Glutathione metabolism and its selective modification. J Biol Chem. 1988;263(33):17205-8.

55. Chen C, Li H, Niu Y, et al. Impact of short-term exposure to fine particulate matter air pollution on urinary metabolome: a randomized, double-blind, crossover trial. Environ Int. 2019;130:104878.

56. Pisoschi AM, Pop A. The role of antioxidants in the chemistry of oxidative stress: a review. Eur J Med Chem. 2015;97:55-74

57. Vignoli A, Tenori L, Luchinat C, Saccenti E. Age and sex effects on plasma metabolite association networks in healthy subjects. J Proteome Res. 2018; 17(1):97-107.

58. Louis P, Flint HJ. Formation of propionate and butyrate by the human colonic microbiota. Environ Microbiol. 2017;19(1):29-41.

59. Yelamanchi SD, Jayaram S, Thomas JK, et al. A pathway map of glutamate metabolism. J Cell Commun Signal. 2016;10(1):69-75.

60. Breitner S, Schneider A, Devlin RB, et al. Associations among plasma metabolite levels and short-term exposure to PM2.5 and ozone in a cardiac catheterization cohort. Environ Int 2016:97:76-84. 
61. Guo Y, Gasparrini A, Armstrong BG, et al. Temperature variability and mortality: a multi-country study. Environ Health Perspect. 2016;124(10):1554-9.

62. Kioumourtzoglou M-A, Spiegelman D, Szpiro AA, et al. Exposure measurement error in PM2.5 health effects studies: A pooled analysis of eight personal exposure validation studies. Environ Health. 2014;13(1):2.

63. Schwartz J, Sarnat JA, Coull BA, Wilson WE. Effects of exposure measurement error on particle matter epidemiology: a simulation using data from a panel study in Baltimore, MD. J Expo Sci Environ Epidemiol. 2007;17(Suppl 2):S2-10.

\section{Publisher's Note}

Springer Nature remains neutral with regard to jurisdictional claims in published maps and institutional affiliations.

Ready to submit your research? Choose BMC and benefit from:

- fast, convenient online submission

- thorough peer review by experienced researchers in your field

- rapid publication on acceptance

- support for research data, including large and complex data types

- gold Open Access which fosters wider collaboration and increased citations

- maximum visibility for your research: over $100 \mathrm{M}$ website views per year

At $\mathrm{BMC}$, research is always in progress.

Learn more biomedcentral.com/submissions 